\title{
Exotic model of the cosmic ray spectrum
}

\author{
Sergey Shaulov ${ }^{1,2, *}$ \\ ${ }^{1}$ P.N.Lebedev Physical Institute of the Russian Academy of Sciences, Moscow, Russia \\ ${ }^{2}$ Moscow Institute of Physics and Technology (State University),MIPT (SU), Moscow, Russia
}

\begin{abstract}
A detailed study of the cores of extensive air showers (EAS) at mountain level (Tien-Shan 690 $\mathrm{g} / \mathrm{cm}^{2}$ ) was carried out in the hybrid experiment HADRON. An analysis of the fine structure of the EAS spectrum in the energy region $10^{15}-10^{17}$ (knee) showed that there are two breaks in the spectrum. Along with the previously known break at an energy of $3 \cdot 10^{15} \mathrm{eV}$, a change in the slope of the EAS spectrum is observed at an energy slightly below $10^{17} \mathrm{eV}$. In addition the use of a large X-ray emulsion chamber (XREC) as a detector of EAS cores allowed us to obtain several new results. An abnormal scaling violation in hadron spectra for $N_{e} \sim 10^{7}\left(E_{0} \sim 10^{16} \mathrm{eV}\right)$ means the existence of a penetrating component of non-nuclear origin. The conclusion about the non-nuclear origin of the penetrating component in the primary radiation of CR is confirmed by the data about the excess of muons in the EAS containing hadrons of maximum energies. It is assumed that the mass composition of primary cosmic radiation varies sharply at energies of $10^{15}-10^{16} \mathrm{eV}$, where quasi-nuclei (strangelets) appear instead of nuclei. A new model of the mass composition of cosmic rays in the region of ultrahigh energies is proposed on this basis .
\end{abstract}

\section{Introduction}

The power spectrum of the primary cosmic radiation (PCR) has two specific areas, the so-called knee at energies of $10^{15}-10^{18} \mathrm{eV}$ and the cutoff of the PCR spectrum at energy $\sim 10^{19}-10^{20} \mathrm{eV}$. Really we are dealing with extensive air showers (EAS) deep in the atmosphere but not with CR. The primary energies of such showers are estimated by means of models of the nuclear cascade using some features of showers. Since $E_{0}$ is a model dependent quantity, we will use the experimental value $N_{e}$ where possible. The EAS spectrum of $N_{e}$ in area of knee has two rather sharp breaks at $N_{e}=10^{6}[1]$ and $N_{e} \sim 10^{8}$ [2]. The origins of these peculiarities of the spectrum are still not clear but often the region between them is considered as the region of the slope adjustment of individual spectra of CR components from protons to iron.

The results of the spectrum measurements of several experiments are shown in figure 1. All spectra are multiplied by $E_{0}^{3}$, allowing to highlight more clearly the presence of inhomogeneities in the all particle spectrum.

The shape of the spectra is different at mountain altitudes and sea level. Figure 2 presents two possible schemes of the EAS spectrum without taking into account the scatter of the data of different experiments. Spectrum 1 is for mountain levels (HADRON and GAMMA) and spectrum 1' for sea level.

The spatial resolution of XREC allows us to allocate individual cascades of $\gamma$-quanta $\left(\pi^{0} \rightarrow 2 \gamma\right)$ and determine individual energies of $\gamma$-quanta with energies above $2 \mathrm{TeV}$. For each cascade in XREC, the angles of arrival $\vartheta$

*e-mail: ser101@inbox.ru

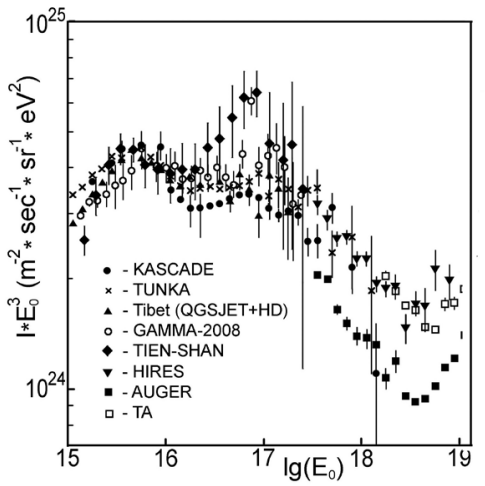

Figure 1. The EAS spectra in different experiments. All spectra are multiplied by $E_{0}^{3}$.

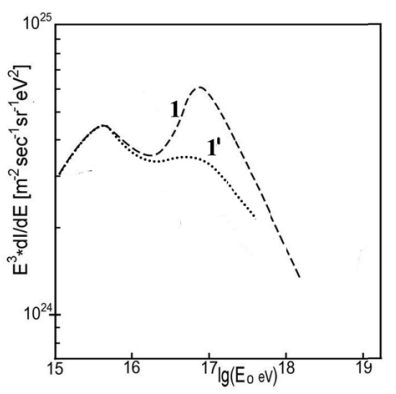

Figure 2. Schematic representation of the EAS spectrum. 1 mountain measurements, 1' for sea level.

and $\varphi$ are determined. Cascades with the same angles are considered to belong to the same shower and are combined 


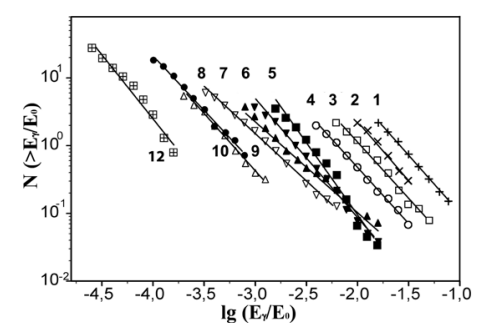

Figure 3. The integral spectra of $x=E_{\gamma} / E_{0}$ in intervals of $N_{e}$ for gamma-families with $\Sigma E_{\gamma} \geq 10 \mathrm{TeV}$.

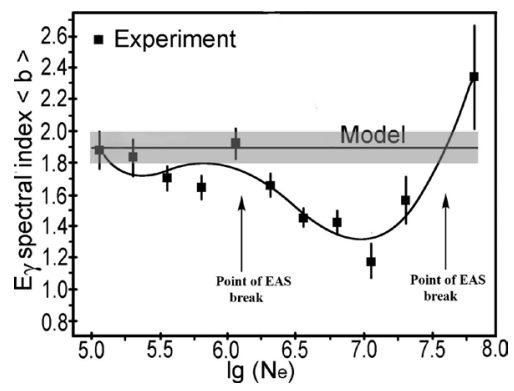

Figure 4. The $N_{e}$ dependence of the slope $\langle b\rangle$ for the integral spectra $N\left(\geq E_{\gamma}\right)$ in gamma-families with $\Sigma E_{\gamma} \geq 10 \mathrm{TeV}$. Horizontal line - the model MQ1n $-\langle b\rangle=1.9 \pm 0.1$.

into a $\gamma$-family. The combination of events in XREC with EAS [3] allows us to construct spectra for a given primary energy and to compare spectra $E_{\gamma}$ in different intervals of primary energies $E_{0}\left(N_{e}\right)$.

The spectra of the most energetic $\gamma$-quanta with $E_{\gamma}>6$ $\mathrm{TeV}$ are shown in figure 3 in different intervals of $N_{e}[4,5]$. It is the integral spectra of $x \geq E_{\gamma} / E_{0}$, where $E_{0}=2 \cdot N_{e}$ $\mathrm{GeV}$ is simple and linear estimation of the EAS primary energy. Representing the spectra of the variable " $x$ " instead of $E_{\gamma}$ avoids them overlapping. The numbers in the figure designate numbers of logarithmic intervals in the range $\lg N_{e}=4.93-7.93$ through $\Delta \lg N_{e}=0.25$ from 1 to 12 . The $\gamma$-quanta with energies more than $6 \mathrm{TeV}$ are absent in the interval at number 11 , which is why this spectrum is missing. The $N_{e}$ dependence of the spectra slopes "b" $\left(\left(N\left(>E_{\gamma}\right) \sim E_{\gamma}^{-b}\right)\right)$ are given in figure 4 compared with the theoretical predictions of the model MQ1n [6].

Figure 3 indicates that the declination of the spectra varies immediately behind the break of the EAS spectrum at $\lg N_{e}=6.1$, and such a correlation is unlikely to be accidental. A $N_{e}$ dependence of the spectral index $<b>$ illustrates that a strong scaling violation takes place in contradiction with model predictions and this violation has an abnormal fashion. An abnormal scaling violation, because of the weighting of cosmic rays in the knee area, would have to lead to a softening of the spectra. The slope of the spectra decreases in the range $N_{e}=10^{6}-10^{7}$, i.e. the spectra become more rigid and the hadron energy increases. Such behavior clearly contradicts the scaling predictions of model MQ1n [8]. By its characteristics, the MQ model is close to the QGSJET (CORSICA) model, since both are based on the quark-gluon string model [7]. The MQ1n

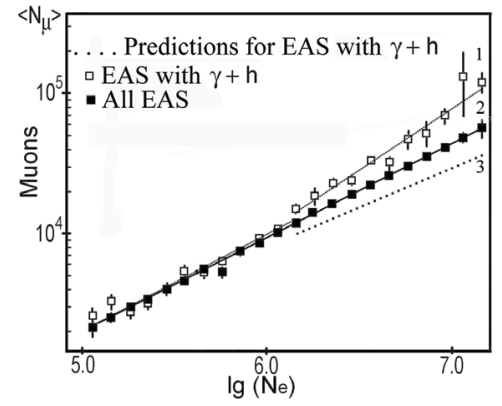

Figure 5. $N_{e}$ dependence of muon number $<N_{\mu}>$.

model assumes the so-called normal mass composition of the PCR with a proton fraction of $40 \%$ at an energy of $3 \cdot 10^{15} \mathrm{eV}$ and the maximum possible scaling violation due to nuclear effects. As follows from the calculations, the slope of the model spectra practically does not change in the knee region.

\section{Discussion}

Of particular interest is the coincidence of the region of scaling violation and restructuring of the $\mathrm{CR}$ spectrum. It means that the changes of the slope of the CR spectrum at region $N_{e}=10^{6}-10^{8}$ should be associated with a violation of scaling in the spectra of EAS hadrons. Let us consider possible explanations for the behavior of the hadronic spectra.

\subsection{Change in the nuclear composition of CR}

A significant increase in the hadron energy at $N_{e} \sim 10^{7}$ means the manifestation of the penetrating component of CR. Among the nuclei it can only be protons. However, it is traditionally believed that the proton spectrum has a break at $N_{e}=10^{6}$ and their contribution to the spectrum of all particles at $N_{e}=10^{7}$ cannot be the main one. Spectrum index "b" close to $b \approx 1$ can be explained if, at $N_{e} 10^{7}$, only protons are present in the CR. It is not possible to explain this situation through any transformation of nuclear spectra.

Formally, the only option that can explain the observed behavior of hadron spectra is as follows. The rigidity of cutoff of the partial nuclear spectra due to their diffusion from the Galaxy or limitation of energy acceleration is $R \sim 10^{14} \mathrm{~V}$. Then the CR spectrum break at $N_{e} \sim 10^{6}$ $\left(E_{0}=3 \times 10^{15} \mathrm{eV}\right)$ corresponds to the break in the partial spectrum of Fe nuclei. For some reason, at high energies, a new nuclear component appears, first of all, protons, etc., to Fe nuclei at $N_{e} \sim 10^{8}$ due to which the hadron spectra are softened in the region $N_{e}=10^{7}-10^{8}$. However, this variant contradicts the data on muons obtained in the HADRON experiment and shown in figure 5.

It shows the $N_{e}$ dependence of the number of muons for all EAS and EAS with $\gamma$-families. The EAS with $\gamma$ families at $\sim 70-80 \%$ are generated by protons and therefore the number of muons should be less than the average 


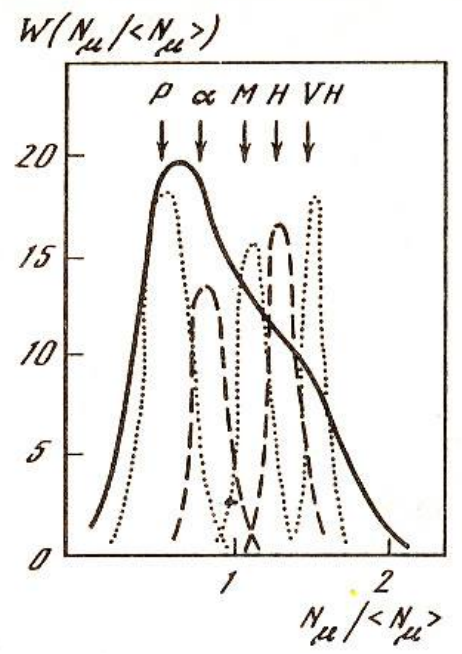

Figure 6. The distributions of $N_{\mu}$ for different primary nuclei and the same $N_{e}$.

for all EAS. This expectation for a mixed nuclear composition of CR is indicated by a dotted line in the figure. The experimental values are higher than the average.

Model distributions of muons in EAS generated by different primary nuclei for the same value of $N_{e}$ are shown in figure 6 [9]. The values of $N_{\mu}$ for light nuclei (p, He) have to be less than the average for all EAS at the same $N_{e}$. The discrepancy between the experimental data and the model could not be eliminated within the framework of nuclear cascades.

No nuclear composition can explain the simultaneous generation of $\gamma$-families and the shown excess of the number of muons. A prominent feature of nuclear cascades is the following, namely, light nuclei generate $\gamma$-rays of high energies, but the number of muons in their EAS have to be relatively low. On the contrary, EAS generated by heavy nuclei should contain many more muons, but do not contain virtually high-energy $\gamma$-rays. Therefore EAS with abnormal rigid $\gamma$-ray spectra and, simultaneously, with a maximal number of muons cannot be explained with traditional nuclear-cascade models. It remains to speculate, that the area of knee is shaped by showers of an unknown origin.

\subsection{Changes in the characteristics of nuclear cascades}

The most naturally sharp form of the break in the EAS spectrum at $N_{e} \simeq 10^{6}\left(E_{0} \sim 3 \mathrm{PeV}\right)$ could be explained by a change in the characteristics of nuclear cascades at a given energy. But there is no model explaining this change. In addition, at $N_{e}=10^{8}$, the slope of the hadron spectrum returns to the previous or slightly larger values of the slope, which can no longer be explained under this hypothesis. Finally, there is no indication in the LHC data of the possibility of any changes to the basic act.

\subsection{A new component of CR?}

If we assume the emergence of a new component in the $\mathrm{CR}$, as follows from the previous considerations, it must be of a non-nuclear origin. Herewith particles in the CR must be stable or metastable with cosmological lifetime of $\tau \sim 10^{6}$ years or more. There is only one such component in theoretical predictions. It is the hypothetical quasinuclei - strangelets [10]. Furthermore we assume that up to $N_{e}=10^{6} \mathrm{CR}$ consist of nuclei, above they consist mainly of strangelets.

Concluding the discussion, we cannot but touch upon the question of why the manifestations of strangelets are not visible in other experiments. The reason apparently is that the experiments with EAS are almost not registering the core of the shower, and the periphery of the EAS is not fundamentally different for nuclear showers and showers formed by strangelets. First of all, this applies to classical ground experiments with detectors of EAS located at large distances from each other. In experiments recording fluorescent light the spatial resolution does not allow to distinguish the contribution of the EAS cores. The most informative in this sense are hybrid experiments using XREC as detector of cores. There are only three such experiments $[5,11,12]$. Information about hadron spectra and the number of muons was obtained only in the experiment HADRON.

\section{The properties of EAS formed by strangelets}

Stranglets are quark bags consisting of a large number of u,d,s-quarks. The baryon number A (baryon charge) for quarks is $1 / 3$. For colorless baryons, which in particular strangelets are, the baryon number is an integer. Therefore, the value of A means the number of triplets of quarks. At baryon numbers $A>10^{3}$ particles of strange quark matter (SQM) can be stable because of a more compact Fermi-Dirac distribution for three quark flavors. In this case, SQM is the basic state of matter. Unlike nuclei, the stability of SQM increases with the baryon number A up to the size of the star. There are three types of SQM: $A<10^{3}$ - unstable particles, $10^{3}<A<10^{7}$ - stable positively charged particles, $A>10^{7}$ - stable neutral particles. Particles with $10^{3}<A<10^{7}$ are called strangelets.

The charge of strangelets is positive, $Z \simeq 0.03 \cdot A$, and on the boundary of a stability $\left(A=10^{3}\right)$, the charge $Z \simeq 30$, which is comparable with the charge of the nucleus Fe. The charge arises because of unequal concentrations of $\mathrm{u}, \mathrm{d}, \mathrm{s}$ quarks at small values of $\mathrm{A}$. The amount of the lightest u-quark is maximal, therefore an electric charge of strangelets is positive and depends on its mass. As A increases, the charge first increases and then decreases when approaching to $A \sim 10^{7}$. The quark concentrations are equalized with baryon number A. For $A \simeq 10^{7}$ the concentrations of u,d,s quarks became equal and the charge $\mathrm{Z}=0$. Due to the charge distribution over a large volume of the strangelet, it can reach values of $Z=1000$. 


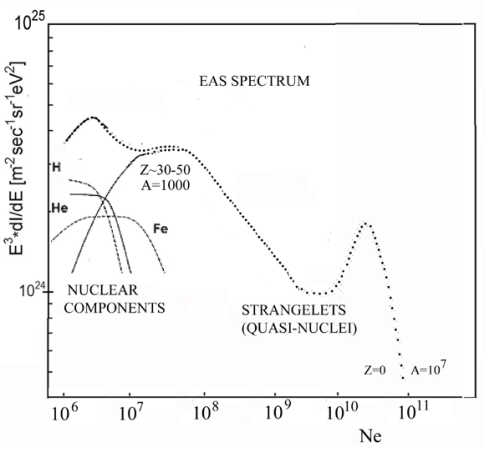

Figure 7. $\mathrm{N}_{e}$ spectra of EAS and individual components of CR.

The properties of the interaction of strangelets with the nuclei of air are qualitatively considered in [13] and partly in [4], using the approach of Bjorken-McLerran [13].

Due to the Coulomb barrier, energy losses are mainly due to the emission of neutral particles $\gamma, \pi^{0}$ and $n$. Due to the large cross-section of interaction and small coefficient of inelasticity, strangelets generate EAS resembling nuclear EAS for the middle group of CNO nuclei [13]. But in some cases the increase in domestic strangeness, $\mathrm{s}$, can lead to instability and decay of strangelet to hundreds of hyperons. Given these properties, we can imagine the following model of the passage of strangelets through the atmosphere.

The penetrating component of the EAS is formed as a result of the decay of the least stable strangelets with A around value $A=10^{3}$ to hundreds of hyperons. This leads to the appearance of rigid hadron spectra at $N_{e} \simeq 10^{7}$. At $N_{e} \sim 10^{8}$, the stability of strangelets increases so much that their decays stop and the spectra of secondary hadrons become softer [4], their slope increases to previous or several large values.

The growth of $N_{e}$ in EAS is due to the increase in the mass of strangelets, not the speed, and the growth of their cross-section of interaction with the size. The spectrum of $N_{e}$ is built according to the increase in the baryon number A of strangelets. The cutoff of the CR spectrum corresponds to $A \simeq 10^{7}\left(N_{e}=10^{10}-10^{11}\right)$ and $Z=0$ and is due the termination of acceleration of strangelets with $Z=0$.

Figure 7 shows the proposed CR spectrum. The basic statement is that above an energy of $10^{17} \mathrm{eV}$ the main component of $\mathrm{CR}$ is strangelets (quasi-nuclei).

Model of strangelets in CR allows to solve a number problems in CR, existing for many years. In addition to the above discussed penetrating component and excess of muons, these are events of type Centauro [13, 14], halo in $\gamma$-families [15], alignment of energetic centers in $\gamma$-families [16], repeated fronts of EAS with large delays of about $100 \mathrm{~ns}$ and more $[17,18]$.

The most reliable test of this model is the measurement of the possible flow of strangelets in outer space for energies $E_{0}>10^{17} \mathrm{eV}$.

\section{Conclusions}

- The CR mass composition includes two different parts: nuclei up to $N_{e} \simeq 10^{6}\left(E_{0}=3 \mathrm{PeV}\right)$ with $A=1-56$ and then strangelets with $A=10^{3}-10^{7}$.

- All CR have galactic origin and the same mechanism of acceleration on the shock waves.

- Cutoff of the CR spectrum is determined by the zero electric charge $\mathrm{Z}$ of strangelets at the maximum baryon number $A \sim 10^{7}$.

\section{References}

[1] Khristiansen G.B., Kulikov G.V., JETPh, 35, 3(9), 635-640, 1958

[2] S.B.Shaulov, Nuclear Physics B (Proc. Suppl.), 122, 2003; arXiv:1610.07427v1 [astro-ph.HE]

[3] S.B.Shaulov, Preprint FIAN, N 245, 3-12, 1987

[4] S.B.Shaulov, Heavy Ion Phys., 4, 403-422,1996

[5] S.B.Shaulov, Cosmic rays about strange quark stars, ISBN: 978-3-659-77433-1, LAP LAMBERT Academic Pablishin, Deutchland, p. 1-129, 2015

[6] A.M. Dunaevsky, M.V. Zimin, BULLETIN de la société des sciences et des lettres de LÓDŹ,série: recherches sur les déformations, XVI, 1994

[7] A. Borisov, V. Denisova, Z. Guseva, A. Iwan, S. Karpova, J.Kempa, A. Krys, V. Maximenko, V. Puchkov and S. Slavatinski, 26th ICRC, Salt Lake City, vol. 1, p. 84-87, 1999

[8] Dunaevsky A.D., Krutikova N.P., Slavatinsky S.A., 22 ICRC, Dublin, 4, p.133-136, 1991

[9] S.I.Nikolsky, Problems of physics of cosmic rays, Nauka, p.169-185, 1987

[10] A. Bodmer, Phys. Rev. D, 4, 1601, 1971

[11] M.Tamada, H.Aoki, K.Honda, N.Inoue, N.Kawasumi, N.Martinic, N.Ochi, N.Ohmori, A.Ohsawa, H.Semba1, and R.Ticona, EPJ Web of Conferences, 52 07006, 2013; DOI: 10.1051/epjconf201/35207006.

[12] Tibet AS Collaboration: M.Amenomori et al., Phys. Rev. D 62, 112002-1, 072007-3, 2000; Phys. Lett. B, 58, p.632, 2006

[13] Bjorken J.D., McLerran L.D., Phys. Rev. D, 20, 2353, 1979

[14] C.M.G. Lattes, Y. Fujimoto and S. Hasegawa, Phys. Rev., 65, p.151, 1980

[15] V.V. Arabkin, V.A. Borodkin, M.D. Smirnova, Yu.A. Smorodin, S.B.Shaulov, 6-th ISVHECRI, Tarbe, p.257-260, 1990; Pamir Collaboration, Izv. AN USSR, ser. fiz., 46, 9, p.1790-1792, 1982

[16] A.S.Borisov, Z.M.Guseva, V.G.Denisova et al., Izv. RAN, ser. fiz., 58, N12, p.10-12, 1994

[17] Jelly J.V., Whitehouse W.J., Proc. Phys. Soc. A, 66, p. 454,1953

[18] D.Beznosko, R.Beisembaev, K.Baigarin, E.Beisembaeva, O.Dalkarov, V.Ryabov, T.Sadykov, S.Shaulov, A.Stepanov, M.Vildanova, N.Vildanov and V.Zhukov, EPJ Web of Conferences 145, 14001, 2017; DOI: 10.1051/epjconf/201714514001 